\title{
Efeitos da pressão positiva contínua em vias aéreas sobre os sintomas nasofaríngeos em pacientes com a síndrome da apnéia obstrutiva do sono*
}

\author{
Effects of continuos positive airway pressure on nasal and pharyngeal symptoms \\ in patients with obstructive sleep apnea
}

\author{
ADELAIDE CRISTINA DE FIGUEIREDO, MARIA CECÍLIA LORENZI, SIMONE PREZZOTI(TESBPT), \\ MARÍLIA MONTENEGRO CABRAL(TESBPT), LUIZ UBIRAJARA SENNES, GERALDO LORENZI-FILHO(TESBPT)
}

Introdução: Sintomas nasofaríngeos são comuns em pacientes com a síndrome da apnéia obstrutiva do sono (SAOS) em tratamento com pressão positiva contínua em vias aéreas (CPAP). No entanto, sintomas nasofaríngeos são também comuns em pacientes com SAOS antes do início do tratamento.

Objetivo: Determinar o impacto do tratamento com CPAP nasal sobre os sintomas nasofaríngeos em pacientes com SAOS.

Método: Foram avaliados 35 pacientes (28 homens), com idade de $54 \pm 10$ anos portadores de SAOS moderada a grave diagnosticada através de polissonografia. Os sintomas nasofaríngeos (espirros, coriza, prurido, obstrução, sangramento e ressecamento nasal e de garganta) foram quantificados através de questionário aplicado antes e depois de pelo menos 3 meses de tratamento com CPAP nasal.

Resultados: 0 índice de apnéia + hipopnéia foi de $50 \pm 25$ eventos por hora. Ao menos um sintoma nasofaríngeo estava presente em 26 pacientes $(74 \%)$ antes do tratamento. A obstrução nasal foi o sintoma mais comum, presente em 18 pacientes $(51 \%)$. Dentre os pacientes inicialmente assintomáticos ( $n=9), 78 \%$ apresentaram alguma reação nasofaríngea adversa com o tratamento. Em contraste, nos pacientes inicialmente sintomáticos, houve redução significativa da intensidade da obstrução, do ressecamento nasal e de garganta e do sangramento nasal após o tratamento.

Conclusões: Sintomas nasofaríngeos são freqüentes em pacientes com SAOS. 0 uso de CPAP pode tanto desencadear sintomas nasofaríngeos em pacientes assintomáticos, como reduzir sua intensidade nos pacientes com sintomas prévios.
Background: Nasal and pharyngeal symptoms are common in patients with obstructive sleep apnoea (OSA) treated with nasal continuous positive airway pressure (CPAP). However, these symptoms are common in OSA patients even before the treatment.

Objectives: Determine the impact of nasal CPAP on nasal and pharyngeal symptoms in OSA patients.

Method: Thirty-five adult patients (28 males), age $54 \pm 10$ years old, with OSA diagnosed by polissonography. All patients answered to a questionnaire about the presence and quantification of sneezing, rhinorrhea, nasal pruritus, obstruction and bleeding, nasal and pharyngeal dryness. The questionnaire was answered before and after at least three months of CPAP therapy.

Results: The apnea-hypopnea index was $50 \pm 25$ events per hour. Twenty six patients (74\%) presented at least one naso-pharingeal symptom before treatment. Nasal obstruction was the most common symptom, being referred by 18 patients (51\%). Among the patients that were initially assymptomatic $(n=9), 78 \%$ developed adverse nasal reactions to CPAP. In contrast, among the patients that presented nasal symptoms before treatment, there was a significant reduction in nasal obstruction, nasal and throat dryness scores as well as nasal bleeding after CPAP therapy.

Conclusions: Nasal na pharyngeal symptoms are frequent in OSAS patients. CPAP therapy may originate nasal symptoms in patients previously assymptomatic, as well as reduce the intensity of these symptoms in patients that are previously symptomatic.

Key words: Obstructive sleep apnea syndrome. Nasal obstruction. Continuous positive airway pressure, compliance.
Descritores: Síndrome da apnéia obstrutiva do sono. Sintomas nasofaríngeos. Obstrução nasal. Máscara de pressão positiva contínua em vias aéreas superiores.

* Trabalho realizado no Laboratório do Sono, Instituto do Coração do Hospital das Clínicas, Disciplina de Pneumologia da Faculade de Medicina da Universidade de São Paulo - FMUSP

Endereço para correspondência - Geraldo Lorenzi-Filho. Av. Dr. Enéas de Carvalho Aguiar, 44 - Laboratório do Sono - 70 andar - Bloco 1 05403-000 - São Paulo - S.P.

Recebido para publicação, em 22/09/03. Aprovado após revisão, em 18/8/04. 


\section{INTRODUÇÃO}

A síndrome da apnéia obstrutiva do sono (SAOS) é definida pela ocorrência de episódios de apnéias e/ou hipopnéias obstrutivas recorrentes durante o sono acompanhadas de sonolência diurna e/ou disfunção cardiovascular ${ }^{(1)}$. Os episódios de apnéia ou hipopnéia presentes nos indivíduos com SAOS são decorrentes do relaxamento fisiológico da musculatura faríngea durante o sono, com conseqüente estreitamento ou obstrução completa das vias aéreas superiores ${ }^{(1)}$. Dados epidemiológicos norte-americanos mostram que $2 \%$ a $4 \%$ dos indivíduos de meia-idade apresentam SAOS ${ }^{(2)}$. Além da grande prevalência, a elevada morbidade observada faz com que a SAOS seja considerada um problema crescente de saúde pública(2).

0 tratamento de escolha para a SAOS moderada e grave é a aplicação noturna de pressão positiva continua nas vias aéreas (CPAP) através do uso de máscara nasa|(1). 0 CPAP nasal virtualmente elimina os eventos respiratórios assim que uma pressão adequada é estabelecida. Apesar de extremamente efetivo, a adesão ao CPAP é variável, sendo a intolerância à mascara um problema clínico comum ${ }^{(3)}$. 0 fluxo contínuo de ar nas vias aéreas superiores pode ocasionar desconforto, muitas vezes associado a queixas nasofaríngeas, em particular ressecamento nasal(4).

Sintomas nasofaríngeos como ressecamento nasal e de garganta, rinorréia e espirros são comuns em pacientes com SAOS, mesmo antes do início do tratamento com CPAP(5). Um dos sintomas mais freqüentes nesses pacientes é a obstrução nasal. Existem teorias que atribuem um papel da obstrução nasal na gênese da $\mathrm{SAOS}^{(6)}$. A obstrução nasal leva a um aumento do esforço inspiratório, com aumento da pressão negativa intraluminal, o que gera instabilidade das vias aéreas superiores e maior tendência ao colapso da faringe ${ }^{(6)}$. Embora os sintomas nasofaríngeos sejam freqüentes em pacientes utilizando $\operatorname{CPAP}^{(6,7)}$, não está claro se esses sintomas já estavam presentes antes do início do tratamento. Brander ${ }^{(5)}$ descreveu aumento de freqüência e intensidade na maioria dos sintomas nasais após o uso do CPAP. Em contraste, Lojander ${ }^{(7)}$ não encontrou alterações significativas na freqüência de sintomas nasais após o tratamento com CPAP.

0 objetivo deste estudo foi determinar o impacto do CPAP nasal sobre as características, a freqüência e a intensidade dos sintomas nasofaríngeos em pacientes com SAOS moderada a grave.

\section{MÉTODO}

Foram estudados 35 pacientes adultos com idade de $54 \pm 10$ anos (média \pm desvio padrão), 28 homens e 7 mulheres, consecutivamente atendidos no Laboratório do Sono do Instituto do Coração do Hospital das Clínicas da Faculdade de M edicina da Universidade de São Paulo. Todos os pacientes realizaram exame de polissonografia completo, que incluiu monitorização contínua de eletroencefalograma ( 2 canais centrais e 2 occipitais), eletromiograma sub-mentoniano e tibial anterior, eletro-oculograma, fluxo aéreo através da cânula nasal e termistor, cintas torácica e abdominal, sensor de ronco, detector de posição e oximetria de pulso.

Foram incluídos apenas pacientes com SAOS moderada a grave, com acesso ao uso de CPAP. Aqueles que não se mantiveram em acompanhamento regular foram excluídos. Uma vez feito o estudo polissonográfico diagnóstico, os pacientes foram submetidos a nova polissonografia com titulação da pressão do CPAP. Seguindo a rotina do laboratório, todos os pacientes fizeram uma sessão de fisioterapia respiratória para adaptação ao CPAP. Nessa ocasião os conceitos básicos do uso do aparelho gerador de pressão positiva foram explicados e os pacientes utilizaram o CPAP com máscara nasal por cerca de 60 minutos. Nessa ocasião (antes do uso do aparelho), foi aplicado um questionário referente à presença $e$ quantificação de sintomas nasofaríngeos. 0 questionário consistia de perguntas sobre espirros, coriza, prurido, ressecamento, obstrução e sangramento nasal. Foi também avaliada a presença de ressecamento de garganta. Cada sintoma foi subjetivamente quantificado pelos próprios pacientes em: (0) ausente; (1) leve: presença esporádica, sem comprometimento de atividades diárias; (2) moderado: sintomas freqüentes com incômodo nas atividades diárias; ou (3) intenso: sintomas constantes, com comprometimento das atividades diárias. Nessa ocasião foi também determinada a sonolência subjetiva através da escala de sonolência de Epworth ${ }^{(8)}$. A escala subjetiva de sonolência de Epworth quantifica, através de um questionário padronizado, a chance de 0 indivíduo cochilar em 8 situações diferentes. A pontuação varia entre 0 e 24, sendo considerado presença de sonolência excessiva valores maiores do que 10 pontos.

Os pacientes foram sistematicamente contactados por telefone (ou através de novas visitas, se necessário) após uma semana, 1 mês e 3 meses após o início do tratamento com CPAP. Conforme a rotina do laboratório, após esse período os pacientes são reavaliados a cada 
6 meses. 0 questionário dos sintomas nasais foi repetido após o uso de pelo menos 3 meses do CPAP em entrevista realizada pessoalmente. Todos os pacientes foram tratados com gerador de fluxo de pressão positiva (CPAP Sullivan ${ }^{\circledR} \mathrm{S} 5$ e $\mathrm{S} 6$ - ResMed ou Solo LX plus ${ }^{\circledR}$ Respironics) com contador de horas de uso (horímetro), que registra 0 número de horas que 0 aparelho funcionou. Foi estimada a adesão do paciente ao CPAP através da determinação do número de horas registrado no aparelho gerador de pressão, dividido pelo número de dias desde 0 início do tratamento. 0 protocolo foi aprovado pelo comitê de ética da instituição.

Foi utilizado o teste $t$ de Student (comparação das médias de idade e indice de apnéia e hipopnéia em pacientes com e sem sintomas nasais). Foi também empregado o teste não-paramétrico de Wilcoxon para a comparação dos escores médios de cada sintoma nasal antes e depois do CPAP. 0 nível de significância considerado foi de $p<0,05$ em testes bi-caudais.

\section{RESULTADOS}

0 índice de apnéia e hipopnéia foi de $50 \pm 25$ eventos por hora de sono, variando entre 24 a 112 eventos por hora. 0 nível de sonolência subjetiva, determinada pela escala de Epworth, foi de $15 \pm 6$. Dentre os 35 pacientes estudados, 26 (74\%) referiam a presença de pelo menos um sintoma naso-faríngeo antes do início do tratamento com CPAP. 0 sintoma mais freqüente foi a obstrução nasal, presente em 18 pacientes $(51 \%)$. Os demais sintomas foram ressecamento de garganta (16 pacientes), ressecamento nasal (10 pacientes), espirros ( 9 pacientes), prurido nasal (7 pacientes), coriza e sangramento nasal (ambos referidos por 6 pacientes). Os pacientes com sintomas nasofaríngeos presentes $(n=26)$ e ausente $(n=9)$ antes do início do tratamento eram semelhantes quanto a idade $(54 \pm 9$ e $55 \pm 11$ anos, respectivamente, $p=0,82$ ) e indice de apnéia hipopnéia ( $39 \pm 25$ e $49 \pm 26$ eventos/ hora, respectivamente, $p=0,34$ ).

No momento da segunda avaliação os pacientes estavam utilizando o CPAP por 5,7 $\pm 1,9$ horas por noite. A pressão de CPAP era de $9,7 \pm 1,5 \mathrm{CmH}_{2} \mathrm{O}$ (variando entre 5 e $13 \mathrm{cmH}_{2} \mathrm{O}$ ). A re-avaliação foi realizada $13 \pm 21$ meses após o início do tratamento. A distribuição dos vários sintomas após CPAP nasal nos pacientes inicialmente assintomáticos, bem como sua intensidade, estão apresentadas na Tabela 1. Dentre os 9 pacientes inicialmente sem sintomas nasofaríngeos, apenas 2 permaneceram assintomáticos após o uso do CPAP. Três pacientes desenvolveram 2 ou mais sintomas equatro pacientes desenvolveram 1 sintoma. Os sintomas mais comuns foram ressecamento nasal, coriza, ressecamento de garganta e obstrução nasal. Sangramento foi observado em apenas um paciente.

A intensidade e evolução dos sintomas nasofaríngeos nos 26 pacientes com algum sintoma inicial estão representadas na Tabela 2. De forma geral, ocorreu uma tendência à redução na intensidade média dos sintomas nasofaríngeos. Foram encontradas reduções estatisticamente significativas na intensidade dos sintomas de obstrução $(p<0,01)$, ressecamento nasal ( $p<0,02)$, sangramento nasal $(p<0,01)$, e ressecamento de garganta $(p<0,01)$. Apesar de uma redução global na intensidade dos sintomas, o efeito do CPAP foi heterogêneo. Em 13 pacientes observouse 0 aparecimento de pelo menos um novo sintoma nasofaríngeo após o uso de CPAP. No caso do ressecamento nasal, por exemplo, ocorreu uma diminuição significativa da intensidade média do sintoma, porém houve aumento do número de pacientes que o referiram após o CPAP (10 pré e 14 pós CPAP) (Tabela 2).

\section{DISCUSSÃO}

A freqüência de sintomas nasofaríngeos em nossa amostra de estudo antes do início do tratamento com CPAP foi alta e semelhante ao descrito previamente na literatura ${ }^{(4,5,7)}$. Encontramos índices próximos a $50 \%$ de obstrução e ressecamento nasal e de garganta. 0 impacto do CPAP sobre os sintomas nasofaríngeos foi extremamente variável. Analisando apenas os pacientes inicialmente assintomáticos, obtivemos reações nasofaríngeas adversas em um número significativo de casos. Entretanto, os efeitos colaterais foram em geral leves. Apesar da observação de uma grande quantidade de problemas nasofaríngeos em pacientes com SAOS em uso de CPAP, os sintomas em geral já estavam presentes antes de seu uso.

O nosso estudo partiu da observação clínica de que muitos pacientes com SAOS apresentam sintomas nasofaríngeos, em particular obstrução nasal. Na maioria das vezes, a não ser que ativamente questionados, os sintomas não são relatados espontaneamente pelo paciente. Essa observação é consistente com o trabalho de Ohki et al ${ }^{(9)}$. Estes autores estudaram a relação entre respiração bucal e obstrução nasal em pacientes com e sem SAOS. Apesar de a resistência nasal ser maior em pacientes com SAOS, a maioria dos pacientes não se queixava de sensação de obstrução nasal. Portanto, apesar de vários pacientes com SAOS passarem a se 
TABELA 1

Ocorrência de sintomas nasofaríngeos adversos após o tratamento com CPAP nasal nos pacientes inicialmente assintomáticos $(n=9)$.

\begin{tabular}{cccccccc}
\hline & $\begin{array}{c}\text { Ressecamento } \\
\text { nasal }\end{array}$ & Coriza & $\begin{array}{c}\text { Ressecamento } \\
\text { garganta }\end{array}$ & Obstrução & Prurido & Espirros & Epistaxe \\
\hline Leve & 1 & 2 & - & 1 & 1 & - & - \\
Moderado & 1 & 1 & 1 & 1 & - & 1 & 1 \\
Intenso & 1 & - & 1 & - & 1 & - & - \\
\hline
\end{tabular}

TABELA 2

Número de pacientes (com sua respectiva porcentagem), intensidade dos sintomas nasofaríngeos antes e depois do uso de CPAP nasal nos 26 pacientes inicialmente sintomáticos.

\begin{tabular}{|c|c|c|c|c|c|c|c|c|c|c|}
\hline \multirow[b]{2}{*}{ SINTOMA } & \multicolumn{5}{|c|}{$\begin{array}{l}\text { PRÉ- CPAP } \\
\text { Intensidade }\end{array}$} & \multicolumn{5}{|c|}{$\begin{array}{l}\text { PÓS- CPAP } \\
\text { Intensidade }\end{array}$} \\
\hline & $\begin{array}{l}\text { Tot } \\
(\%)\end{array}$ & $\mathbf{L}$ & M & I & $\begin{array}{l}\text { Escore } \\
\text { médio } \\
\text { (Med) }\end{array}$ & $\begin{array}{l}\text { Tot } \\
(\%)\end{array}$ & $\mathbf{L}$ & $M$ & I & $\begin{array}{l}\text { Escore } \\
\text { médio } \\
\text { (Med) }\end{array}$ \\
\hline $\begin{array}{l}\text { Obstrução } \\
\text { nasal }\end{array}$ & $\begin{array}{c}18 \\
(51 \%)\end{array}$ & 6 & 6 & 6 & $\begin{array}{c}2,0 \\
(2,0)\end{array}$ & $\begin{array}{l}14 \\
(40 \%)\end{array}$ & 8 & 5 & 1 & $\begin{array}{l}1,0^{*} \\
(1,0)\end{array}$ \\
\hline $\begin{array}{l}\text { Ressecamento } \\
\text { de garganta }\end{array}$ & $\begin{array}{l}16 \\
(46 \%)\end{array}$ & 4 & 4 & 8 & $\begin{array}{c}2,3 \\
(2,5)\end{array}$ & $\begin{array}{l}14 \\
(40 \%)\end{array}$ & 9 & 3 & 2 & $\begin{array}{c}0,7 * \\
(1,0)\end{array}$ \\
\hline $\begin{array}{l}\text { Ressecamento } \\
\text { nasal }\end{array}$ & $\begin{array}{l}10 \\
(29 \%)\end{array}$ & 1 & 3 & 6 & $\begin{array}{c}2,4 \\
(3,0)\end{array}$ & $\begin{array}{l}14 \\
(40 \%)\end{array}$ & 9 & 4 & 1 & $\begin{array}{c}0,8 * \\
(1,0)\end{array}$ \\
\hline Espirros & $\begin{array}{c}9 \\
(26 \%)\end{array}$ & 2 & 4 & 3 & $\begin{array}{c}2,0 \\
(2,0)\end{array}$ & $\begin{array}{l}11 \\
(31 \%)\end{array}$ & 6 & 2 & 3 & $\begin{array}{c}1,3 \\
(1,0)\end{array}$ \\
\hline Prurido & $\begin{array}{c}7 \\
(20 \%)\end{array}$ & 2 & 2 & 3 & $\begin{array}{c}2,0 \\
(2,0)\end{array}$ & $\begin{array}{c}9 \\
(26 \%)\end{array}$ & 8 & 0 & 1 & $\begin{array}{c}1,0 \\
(1,0)\end{array}$ \\
\hline Coriza & $\begin{array}{c}6 \\
(17 \%)\end{array}$ & 2 & 3 & 1 & $\begin{array}{l}1,9 \\
(2,0)\end{array}$ & $\begin{array}{l}10 \\
(29 \%)\end{array}$ & 6 & 4 & 0 & $\begin{array}{c}0,7 \\
(1,0)\end{array}$ \\
\hline Epistaxis & $\begin{array}{c}6 \\
(17 \%)\end{array}$ & 4 & 1 & 1 & $\begin{array}{c}1,5 \\
(1,0)\end{array}$ & $\begin{array}{l}2 \\
(5 \%)\end{array}$ & 1 & 1 & 0 & $\begin{array}{c}0,0 * \\
(0,0)\end{array}$ \\
\hline
\end{tabular}

relação ao número total de paciente intenso; Cada escore é representado pela sua média e mediana (Med), $* 0<0,05$ quando 0 escore médio de sintomas pós CPAP foi significativamente menor do que o escore médio inicial.

queixar do sintoma nasal após o uso do CPAP, muitas vezes o sintoma já estava presente antes do início do tratamento $0^{(7)}$.

Estudos populacionais sugerem que existe uma relação entre obstrução nasal e SAOS. Numa análise populacional de 5000 pacientes, Young et a/(10) constataram que pacientes com sintomas de obstrução nasal apresentavam maior chance de apresentar ronco e sonolência excessiva diurna. Adicionalmente, os pacientes com obstrução nasal devida a alergia tinham uma chance 1,8 vezes maior de apresentar SAOS moderada a grave. Existem ao menos dois mecanismos pelos quais a obstrução nasal pode contribuir com a gênese da SAOS. A obstrução nasal leva a um aumento do esforço inspiratório, com aumento da pressão negativa intraluminal, gerando instabilidade das vias aéreas superiores e maior tendência ao colapso da faringe ${ }^{(4)}$. Além disso, a obstrução nasal pode levar à respiração bucal que, durante o sono, representa um aumento significativo na resistência das vias aéreas superiores conforme demonstrado por Fitzpatrick e colaboradores $^{(11)}$ Maurice et al (12) demonstraram que a abertura da boca em indivíduos normais aumenta a tendência ao colapso das vias aéreas durante o sono e pode contribuir para a SAOS. Por outro lado, Miljeteig et $a l^{(13)}$ não encontraram qualquer correlação entre 0 
grau de resistência nasal e a gravidade da SAOS. Foi recentemente postulado que a obstrução nasal não é o principal fator envolvido na gênese da SAOS, mas funciona como um importante co-fator ${ }^{(14,15)}$. Independentemente do real significado da obstrução nasal na gênese da SAOS, ela é comum e quando presente em intensidade leve a moderada não impede o tratamento adequado com o CPAP nasal.

Em nosso estudo, como o objetivo era determinar o impacto do CPAP nasal sobre os sintomas nasofaríngeos, incluímos somente pacientes com SAOS moderada a grave. Como o CPAP não é pago pelo governo ou pelos seguros de saúde privados no Brasil, os pacientes compraram o aparelho e provavelmente representam uma amostra de pacientes altamente motivados em relação ao seu uso. Outra limitação importante de nosso estudo é que incluímos apenas pacientes em acompanhamento regular. Isso pode justificar a alta adesão ao CPAP em nossa amostra. Portanto, por termos estudado uma população selecionada, não podemos fazer qualquer inferência do impacto da obstrução nasal sobre a adesão ao tratamento. Embora pareça óbvio que a obstrução nasal deve dificultar a adaptação do CPAP nasal, alguns autores não encontraram relação entre adesão ao CPAP e obstrução nasal ${ }^{16}$. Lojander et $\mathrm{a}^{\Gamma}$ encontraram que a freqüência dos sintomas nasofaríngeos antes e durante o tratamento com CPAP foi similar nos pacientes que pararam o tratamento em comparação com os pacientes que o continuaram.

Um dado interessante em nosso estudo, que é consistente com a observação clínica, éo de que muitos pacientes tiveram uma diminuição na intensidade dos sintomas nasofaríngeos após o tratamento com CPAP. Apesar de um aumento discreto na porcentagem de pacientes que referiam sintomas nasais após o uso de CPAP, a intensidade média dos sintomas de ressecamento nasal, de garganta e epistaxe apresentou queda significativa após o início do tratamento. A nossa hipótese é de que vários pacientes com SAOS são respiradores bucais e que com o uso do CPAP nasal, passam a respirar pelo nariz durante o sono. A respiração nasal restituiria a fisiologia normal das vias aéreas superiores e poderia contribuir para a redução da intensidade de sintomas nasofaríngeos.

Concluímos que a evolução dos sintomas nasofaríngeos após o tratamento com CPAP é variável nos pacientes com SAOS. O uso do CPAP tanto pode desencadear sintomas em pacientes assintomáticos antes do tratamento como pode reduzir sua intensidade em pacientes inicialmente sintomáticos. Apesar da elevada ocorrência de sintomas nasofaríngeos em pacientes com SAOS em tratamento com CPAP, é difícil atribuir esses sintomas a esse tratamento.

\section{REFERÊNCIAS}

1. Guilleminault C; Van der Hoed J, Mitler MM. Clinical overview of the sleep apnea syndrome. In: Guilleminault C, Dement WC, eds. Sleep Apnea Syndrome New Yourk: Ar Liss, 1978; 1-12.

2. Young, T; Peppard, P E; Gottlieb, D J. Epidemiology of Obstructive Sleep Apnéia. Am J Respir Crit Care Med 2002; 165 : 1217-39.

3. Pépin J L, Leger $P$, Veale $D$, Langevin $B$, Robert $D$, Lévy $P$. Side Effects of Nasal Continuous Positive Airway Pressure in Sleep Apnea Syndrome. Chest 1995; 107 : 375-81.

4. Kalan A, Kenyon GS, Seemungal TAR, Wedzicha JA. Adverse Effects of Nasal Continuous Positive Airway Pressure Therapy In Sleep Aonoea Syndrome. J Laryngol Otol 1999;113:888-92.

5. Brander PE, Soirinsuo M, Lohela P. Nasopharyngeal Symptoms in Patients with Obstructive Sleep Apnea Syndrome. Respiration 1999; 66: 128-35.

6. Mirza, N; Lanza, D C. The Nasal Airway and Obstructed Breathing During Sleep. Otolaryngol Clin North America 1999; 32 : 243-63.

7. Lojander J, Brander PE, Ämmälä K. Nasopharyngeal Symptoms and Nasal Continuous Positive Airway Pressure Therapy in Obstructive Sleep Apnoea Syndrome. Acta Otolaryngol (Stockh) 1999; 119 : 497-502.

8. Johns, M W. A new method for measuring daytime sleepiness: The Epworth Sleepiness Scale. Sleep 1991; 14:540-5.

9. Ohki M, Usui N, Kanazawa H. Relationship between oral breathing and nasal obstruction in patients with obstructive sleep apnea. Acta Otolaryngol Suppl (Stockh) 1996; 523:228-30.

10. Young T, Finn L, Kim H. Nasal obstruction as a risk factor for sleep-disordered breathing. J Allergy Clin Immunol, 1997; 99:S757-62.

11. Fitzpaatrick MF, McLean H, Urton, AM, Tan A, O'Donnell d. Driver HS. Effect of Nasal or oral Breathing Rout on Upperr Airway Resistance during Sleep. Eur Respir J 2003; 22:827-32.

12. Maurice J C, Marc I, Carrier G. Effects of mouth opening on upper airway collapsability in normal sleeping subjects. Am J Respir Crit Care Med, 1996; 153: 255-9.

13. Miljeteig $H$, Savard $P, M$ ateika $S$. Snoring and nasal resistance during sleep. Laryngoscope, 1993; 103: 918-23.

14. Friedman M, Tanyeri H, Lim JW, Landsberg R, Vaidyanathan K, Caldarelli D. Effect of Improved Nasal Breathing on Obstructive Sleep Apnea. Otolaryngol Head Neck Surg 2000; 122: 71-4.

15. Miyazaki S, Itasaka Y, Ishikawa K, Togawa K. Influence of Nasal Obstruction on Obstructive Sleep Apnea. Acta Otolaryngol (Stock) 1998; Suppl 537: 43-6.

16. Schechter GL, Ware C, Perlstrom J, McBrayer RH. Nasal Patency and the Effectiveness of Nasal Continuous Positive Air Pressure in Obstructive Sleep Apnea. Otolaryngol Head Neck Surg 1998; 118 : 643-7. 